\title{
Tune Measurement Methods of the Tevatron
}

\author{
C.Y. Tan, X.L. Zhang, P. Lebrun \\ FNAL, Batavia, IL 60510, USA*
}

\begin{abstract}
We will discuss several methods for measuring the tunes in the Tevatron. These methods can be separated into three classes: active, passive and hybrid. In the active method, the beam is tickled in order to obtain a frequency response. In the passive method, a Schottky detector which uses a resonant stripline is used to measure the Schottky spectrum of the beam. In the hybrid method, we tickle the beam using kickers, or the Tevatron Electron Lens (TEL) in order to bring the tune signal above the noise floor of the Schottky detectors. An automatic tune fitting algorithm is also under development which allows us to measure the tune without human intervention.
\end{abstract}

\section{INTRODUCTION}

There are several tune measurement methods used in the operation of the Tevatron. These methods are developed in part because the antiproton tune is difficult to measure when there are protons in the Tevatron. In high energy physics conditions, the proton beam current is about 10 times higher than the antiproton beam current and thus the proton signal swamps the Schottky detectors despite their directivity. The preferred method for measuring proton tunes is to use the Schottky detectors which are passive and thus do not influence the beam in any way. For measuring antiproton tunes, we have to gently excite the beam transversely either with stripline kickers or with the Tevatron Electron Lens (TEL). Finally, a tune fitting method is also under development which will allow us to read the proton tune without human intervention. This method when fully operational will allow us to quickly measure chromaticities and also track the evolution of the proton tunes during collisions.

\section{SCHOTTKY DETECTORS}

The Schottky detectors [1] are $1 \mathrm{~m}$ in length with a square cross section shown in Figure 1. The stripline consists of two copper bars which can be moved by stepper motors to change the sensitivity. Its resonant frequency can also be tuned by using the variable capacitor $C_{v}$. Presently these detectors are tuned to resonate around $21.4 \mathrm{MHz}$ with a loaded $Q_{L}=370$.

Four of these detectors have been built to allow us to look at the horizontal and vertical tunes of both the protons and pbars. However in practice, only the proton tunes are monitored during high energy physics (HEP). Although

\footnotetext{
* Work supported by the U.S. Department of Energy under contract No. DE-AC02-76CH03000.
}

these detectors are directional, the pbar tunes are not normally monitored because alot of the proton signal still leaks through which swamps the pbar signals. In order to overcome this, we have to gently kick - tickle the pbars in order to see their tunes.

\section{Tickling}

The process of gently kicking the beam is what we call tickling. We have two tickling methods which we use to observe pbar tunes using the Schottky detectors

- Tevatron Electron Lens (TEL).

- Gated noise on a stripline kicker.

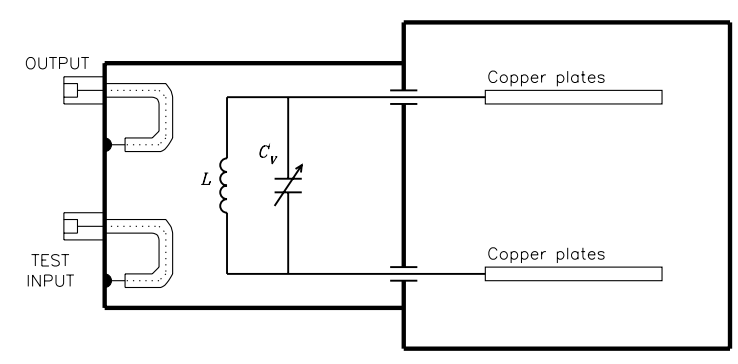

Figure 1: Transverse geometry of the Schottky pickup reproduced from [1]

\section{Tevatron Electron Lens}

The TEL, is built for the Tevatron Beam-Beam Compensation R\&D Project, can also be used as a beam exciter for beam tune measurements [2]. To do this, the electron beam of the TEL is placed very near the bunch to be measured by timing the electron beam pulse to a specific bunch or bunches.

The electron beam used for this purpose is round with a diameter of about $3.5 \mathrm{~mm}$ and is modulated with gated white noise whose modulation depth is also adjustable. Every time the selected bunch passes by the electron beam, it receives a kick from the electron beam (this is just like the stripline beam tickler discussed below). Therefore, beam dipole oscillations are excited and thus Schottky signals which were originally too weak to be seen by a spectrum analyzer is excited above the noise floor. Besides using the white noise modulation, a chirp or a modulation sweep can also be applied. One caveat is that the beam-beam kick can be very strong, and so the peak electron current and modulation depth must be well controlled to avoid fast beam blowups or even beam loss. 
In practice, the electron beam is gated to a specific antiproton bunch and then set to about 1 to $2 \mathrm{~mm}$ from it. This allows us to tickle it and measure its tune. As a comparison, the nearest proton bunch is about $7 \mathrm{~mm}$ away from the electron beam.

\section{Gated Noise Tickling}

In gated noise tickling, a bandwidth limited white noise is applied to one selected pbar bunch with a stripline kicker. Figure 2, shows what happens when we do this to pbar bunch 24 . Before applying the tickle, we see a proton signal which leaks through. After we apply a vertical tickle, we can clearly see the pbar vertical tune.

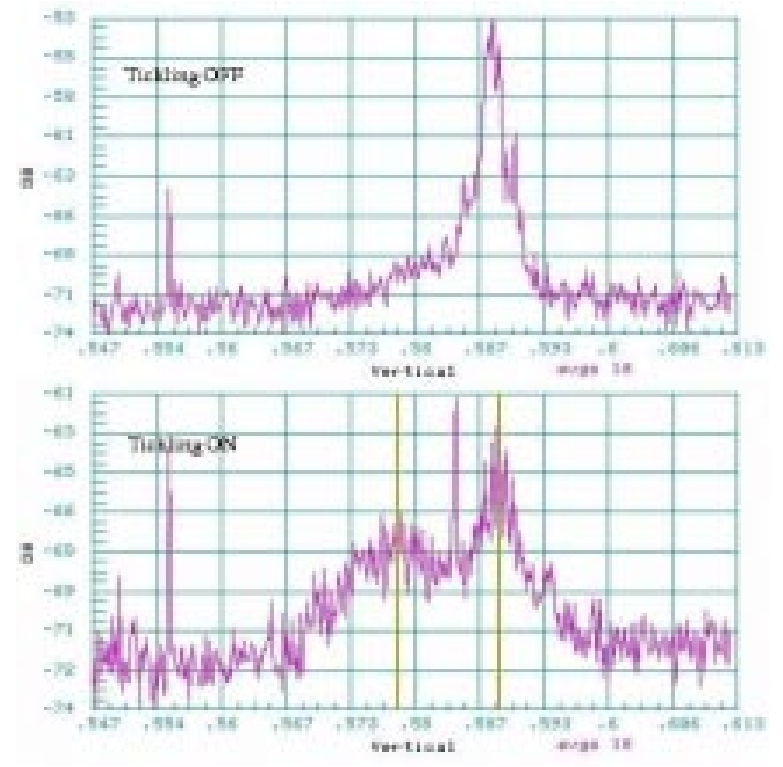

Figure 2: Pbar tune of bunch 24 with gated tickling $\mathrm{ON}$ and OFF

\section{FREQUENCY RESPONSE MEASUREMENT}

We measure the frequency response of each pbar bunch by tickling the beam with a stripline kicker and then measuring the frequency response with a stripline pickup. See Figure 3. A critical part of the setup is the autozero box [3] which centres the beam virtually between the stripline pickups. The sample and hold circuit allows us to measure the pbar tune of individual bunches. Figure 4 shows the frequency response of one pbar bunch.

\section{TUNE FITTING}

In order to automate the tunes, decoupling and chromaticity measurements, we have written a tune fitting software package. Frequency spectra similar to the one shown in Figure 2 are read out at a frequency of approximately $0.5 \mathrm{~Hz}$ into a conventional computer running SUNOS.

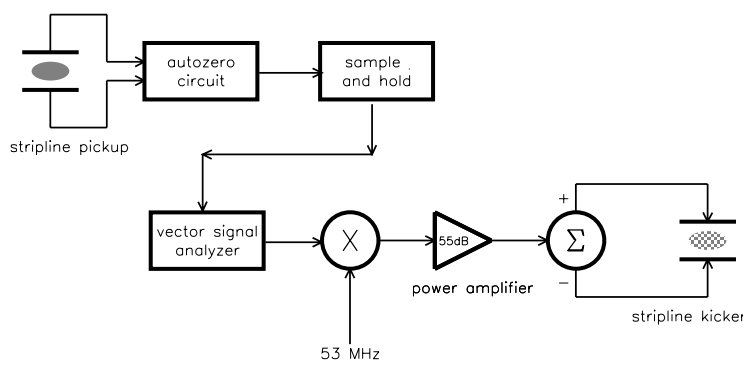

Figure 3: Frequency response measurement setup

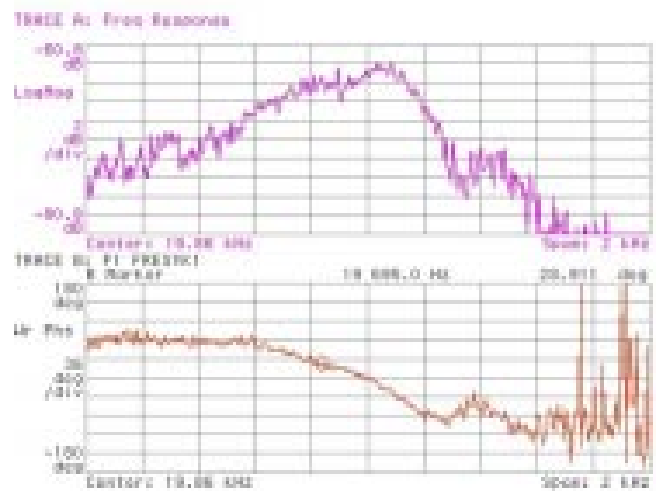

Figure 4: Frequency response of pbar bunch 1

First, such spectra are Gaussian-smeared ${ }^{1}$ to smooth out the data and to select the location of the broad componenents of the spectrum. Only two such peaks are crudely located, corresponding to the vertical and horizontal tunes from the proton beam. Second, each of these regions are analyzed seprately, based on the original data. We look for the characteristic pattern corresponding to the excitation of the synchrotron-betatron $(\mathrm{SB})$ sidelines, separated from the main line frequency by $\approx 85 \mathrm{~Hz}$ and $\approx 31 \mathrm{~Hz}$ at 150 and $980 \mathrm{GeV}$, respectively. We fit the data with an incoherent sum of five resonant "Breit-Wigner" functions:

$$
A(f)=B+\sum_{i=1}^{5}\left(A_{i} \Gamma /\left(\left(f-f_{0, i}\right)^{2}+\Gamma^{2} / 4\right)\right.
$$

where $f_{0}$ is the frequency of the main line, $f_{0,(i+1)}-f_{0, i}$ is the SB tune split, $\Gamma$ is the width of the resonance. The strength of each individual line $A_{i}$ and $B$ the noise floor are free parameters. The tune is located at the line closest to the position of the broad, smoothed-out, peak. Such fits are done on a linear scale to enhance such features.

Figure 5 summarizes these operations. It should be noted that the software often fails to properly assign the correct plane ( $\mathrm{X}$ vs $\mathrm{Y}$ ) for a given set of tunes lines. This is because the Tevatron is strongly coupled (with a typical mini-

\footnotetext{
${ }^{1}$ The value of a given bin in the frequency domain is replaced by the sum of the neighboring bins, weighted by a Gaussian centered in that given bin.
} 
mum tune split of $\sim 0.004$ to $\sim 0.008$ ) and we are running with tune differences in the $\mathrm{X}$ and $\mathrm{Y}$ planes close to this minimum tune split. Maybe a closer look at the relative signal strength on the $\mathrm{X}$ and $\mathrm{Y}$ pick will help sort this out. However, this does not prevent us from measuring tunes or chromaticities for a given plane when we run close to the minimum tune split, because we can keep track of the tunes for each individual plane with past measurement which are taken away from the minimum tune split region.

This method works very well when we tickle the "uncoalesced" (53 MHz bunched beam, with a longitudinal emittance of a fraction of an $\mathrm{eV} \cdot \mathrm{sec}$ ) proton beam. It allows us to accurately measured the betatron coupling by simply sweeping the vertical base tune setting and recording these tunes, as shown in Figure 6. These frequency spectra are more difficult to analyze when relatively large emittance, coalesced (396 ns bunch spacing), and quiescent beam circulates in the Tevatron during HEP operation. Consequently, the fit often fails to locate the SB features. Yet, based on a large number of such fits, stable tunes can be assigned to the proton beam for both planes.

This tune fitter reports results continuously at about 0.5 to $1 \mathrm{~Hz}$, with a delay time of about 5 seconds. This delay is due to the scanning and averaging in the HP3561a spectrum analyzer, about a second or so for Data Acquisition via the GPIB and various computers, and sending the output to the datalogger nodes, and finally about a second for doing the fits. We are currently working on faster algorithms, relying solely on the smoothed out spectra, to speedup this procedures. In addition, we can merge this software with the control software regulating the tickling of specific bunches allowing us to systematically explore quatitatively the beam-beam tune-shift phenomenon in the Tevatron.
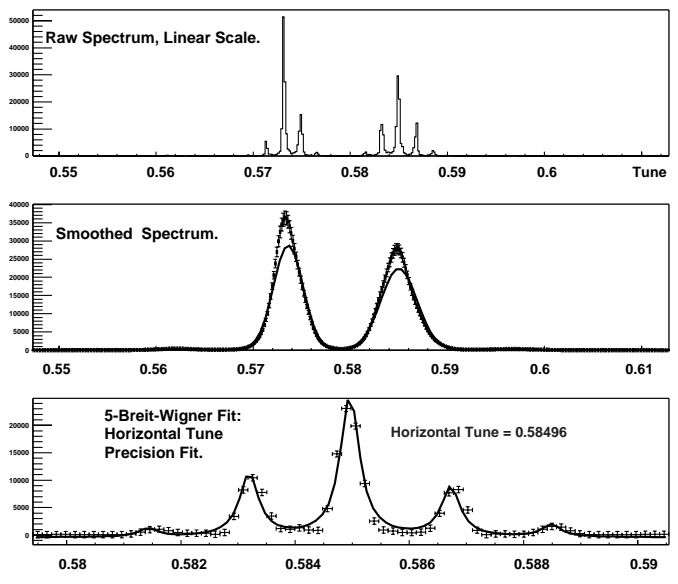

Figure 5: Tune fitter spectra. The vertical scale is related to power, in arbitrary units. The horizontal tune scale is fixed by the known revolution frequency and the analyzer scan settings. Fits are described in the text.

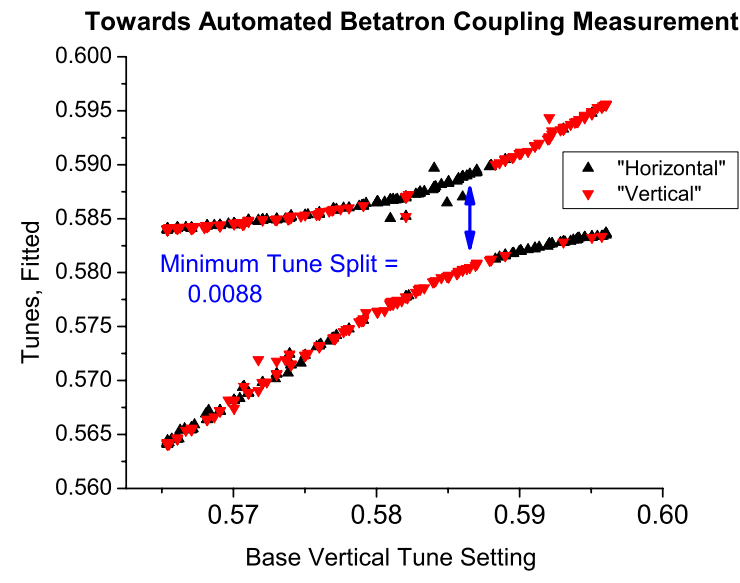

Figure 6: A measurement of the betatron Tune coupling based on the fits discussed above.

\section{CONCLUSION}

We have discussed the various methods which we use to measure the proton and antiproton tunes in the Tevatron. None of the methods for measuring the antiproton tunes are completely satisfactory at this time. A new set of Schottky detectors operating at $1.6 \mathrm{GHz}$ is being commissioned which will allow us to measure bunch by bunch antiproton tunes passively. Unfortunately, the tune resolution of this system will be $\sim 0.005$ rather than $\sim 0.0001$ of the $21.4 \mathrm{MHz}$ Schottky system discussed above. The tune fitting programme also shows promise in providing us with a way of automatically reading and tracking the evolution of the tune during a high energy physics operations.

\section{ACKNOWLEDGMENTS}

We thank John Marrafino for his technical expertise and advices on the fitting software. We also are thankful to members of the Accelerator Controls Deptartment for their data acquisition work and dedicated support.

\section{REFERENCES}

[1] D. Martin, et al, "A Resonant Beam Detector for Tevatron Tune Monitoring", Accelerator Science and Technology Proceedings, vol 3 1486-1488, 1989.

[2] X.Zhang, K.Bishofberger, V.Shiltsev, F.Zimmermann, The Special Applications of Tevatron Electron Lens in Collider Operation, this conference.

[3] C.Y. Tan, "Theory of the Autozero Box and the Transverse Tune Measurement System”, Fermilab, TM-2078, Oct 1999. 